\title{
Development of Core-Fucose-Deficient Humanized and Chimeric Anti-Human Podoplanin Antibodies
}

\author{
Mika K. Kaneko,, Tomokazu Ohishi, ${ }^{2}$ Takuro Nakamura, ${ }^{1}$ Hiroyuki Inoue, ${ }^{2}$ Junko Takei, \\ Masato Sano, Teizo Asano, Yusuke Sayama, Hideki Hosono,, Hiroyoshi Suzuki, ${ }^{1}$ \\ Manabu Kawada, ${ }^{2}$ and Yukinari Kato ${ }^{1,4}$
}

Podoplanin (PDPN), a 36-kDa type I transmembrane $O$-glycoprotein, is expressed in normal cells, including renal epithelial cells (podocytes), lymphatic endothelial cells, and pulmonary type I alveolar cells, and in cancer cells, including brain tumors and squamous cell lung carcinomas. PDPN activates platelet aggregation by binding to C-type lectin-like receptor-2 (CLEC-2) on platelets, and PDPN/CLEC-2 interaction facilitates blood/lymphatic vessel separation. We previously produced an anti-human PDPN monoclonal antibody (mAb), clone NZ-1 (rat $\mathrm{IgG}_{2 \mathrm{a}}$, lambda) and its rat-human chimeric mAbs (NZ-8/NZ-12), which neutralize PDPN/CLEC-2 interactions and inhibit platelet aggregation and cancer metastasis. In this study, we first developed a humanized anti-human PDPN $\mathrm{mAb}$, named as NZ-27. We further produced a core-fucose-deficient version of NZ-27, named as P1027 and a corefucose-deficient version of NZ-12, named as NZ-12f. We investigated the binding affinity, antibody-dependent cellular cytotoxicity (ADCC), complement-dependent cytotoxicity (CDC), and antitumor activity of P1027 and NZ-12f. We demonstrated that the binding affinities of P1027 and NZ-12f against LN319 (a human glioblastoma cell line) are $1.1 \times 10^{-8}$ and $3.9 \times 10^{-9} \mathrm{M}$, respectively. ADCC reporter assays demonstrated that NZ-12f shows 1.5 times higher luminescence than P1027. Furthermore, NZ-12f showed 2.2 times higher ADCC than P1027, whereas both P1027 and NZ-12f showed high CDC activities against LN319 cells. Using LN319 xenograft models, P1027 and NZ-12f significantly reduced tumor development in an LN319 xenograft model compared with control human IgG. Treatment with P1027 and NZ-12f may be a useful therapy for patients with PDPN-expressing cancers.

Keywords: PDPN, humanized antibody, P1027

\section{Introduction}

A 36-KDA TYPE I TRANSMEMBRANE $O$-glycoprotein, podoplanin (PDPN)/Aggrus/T1 $\alpha / g p 36$, is a platelet aggregation-inducing factor. It is expressed in many cancers, including malignant brain tumors, mesothelioma, and several squamous cell carcinomas. ${ }^{(1-10)}$ Expression of PDPN is associated with cancer metastasis, epithelial-mesenchymal transition, malignant progression, ${ }^{(11-18)}$ and clinical outcome. ${ }^{(19-21)}$ Only a small and phenotypically distinct subset of cells in solid tumors are considered tumor-initiating cells (TICs) that are resistant to conventional therapies and responsible for relapse. ${ }^{(2)}$ Therefore, targeting TICs could be useful for cancer therapy. ${ }^{(23)}$ PDPN is reported to be a
TIC marker ${ }^{(24)}$; thus, immunotherapy using specific antibodies against PDPN may eradicate TICs in cancers.

We previously produced a rat anti-human PDPN monoclonal antibody (mAb), NZ-1. ${ }^{(5)} \mathrm{NZ}-1$ is highly specific and sensitive, and displays high binding affinity against PDPN. ${ }^{(25,26)}$ NZ-1 inhibited tumor cell-induced platelet aggregation and cancer metastasis. ${ }^{(12)}$ NZ-1 is highly internalized into glioma cells, and is well accumulated into tumors in vivo, making NZ-1 a suitable candidate for therapy against malignant gliomas. ${ }^{(27)} \mathrm{We}$ constructed a single-chain antibody variable region fragment using NZ-1 (NZ-1-scFv). ${ }^{(28)}$ NZ-1-scFv was then fused to Pseudomonas exotoxin A (NZ-1-scFv-PE38). NZ-1-scFv-PE38 demonstrated significant activity against glioblastoma and medulloblastoma

${ }^{1}$ Department of Antibody Drug Development, Tohoku University Graduate School of Medicine, Sendai, Japan.

${ }^{2}$ Institute of Microbial Chemistry (BIKAKEN), Numazu, Microbial Chemistry Research Foundation, Numazu-shi, Japan.

${ }^{3}$ Department of Pathology and Laboratory Medicine, Sendai Medical Center, Sendai, Japan.

${ }^{4}$ New Industry Creation Hatchery Center, Tohoku University, Sendai, Japan.

(c) Mika K. Kaneko et al. 2021; Published by Mary Ann Liebert, Inc. This Open Access article is distributed under the terms of the Creative Commons License (http://creativecommons.org/licenses/by/4.0), which permits unrestricted use, distribution, and reproduction in any medium, provided the original work is properly cited.

Correction added on February 17, 2021 after first online publication of October 21, 2020; The article reflects Open Access, with copyright transferring to the author(s), and a Creative Commons License (CC-BY) added (http://creativecommons.org/licenses/by/4.0). 
cells, and induced tumor growth delay in D2159MG and D283MED using in vivo tumor models.

We previously produced rat-human chimeric anti-PDPN antibodies (NZ-8 and NZ-12) from NZ-1. ${ }^{(29,30)}$ Light chains of NZ-8 and NZ-12 are human kappa and human lambda, respectively. NZ-12 possesses higher antibody-dependent cellular cytotoxicity (ADCC) and complement-dependent cytotoxicity (CDC) compared with NZ-8. ${ }^{(30)} \mathrm{NZ}-12$, together with human natural killer (NK) cells, showed antitumor effects against malignant pleural mesothelioma orthotopic xenograft mice. ${ }^{(31)}$ Furthermore, radioimmunotherapy (RIT) with ${ }^{90}$ Y-labeled NZ12 significantly suppressed tumor growth in an NCI-H226 mesothelioma xenograft mouse model, prolonging survival without loss of body weight or obvious adverse effects. ${ }^{(32)}$

Many studies have revealed that core-fucose-deficient antibodies are known to show higher ADCC activities. ${ }^{(33,34)}$ Similarly, we previously produced a core-fucose-deficient version of anti-podocalyxin $\mathrm{mAb}\left(47-\mathrm{mG}_{2 \mathrm{a}}\right)$, which was named as $47-\mathrm{mG}_{2 \mathrm{a}}-\mathrm{f}$, to augment its ADCC activities. ${ }^{(35)}$ As expected, 47- $\mathrm{mG}_{2 \mathrm{a}}-\mathrm{f}$ exhibited stronger ADCC activities than $47-\mathrm{mG}_{2 \mathrm{a}}$ against OSCC cell lines such as HSC-2 and SAS cells, leading to higher antitumor activities in those xenograft models. Furthermore, we recently developed a core-fucosedeficient mouse-canine chimeric anti-dog PDPN mAb (P38B), named as P38Bf. ${ }^{(36)}$ We compared ADCC between P38B and P38Bf against Chinese hamster ovary $(\mathrm{CHO}) / \mathrm{dPDPN}$ cells. Although both $\mathrm{P} 38 \mathrm{~B}$ and $\mathrm{P} 38 \mathrm{Bf}$ revealed high ADCC activities against $\mathrm{CHO} / \mathrm{dPDPN}$ cells, $\mathrm{P} 38 \mathrm{Bf}$ demonstrated significantly higher ADCC compared with $\mathrm{P} 38 \mathrm{~B}$, especially at low concentrations, indicating that core-fucose-deficient mAbs are very useful for increasing ADCC activities.

In this study, we produced a core-fucose-deficient version of a humanized anti-human PDPN mAb (NZ-27), named as P1027 and a core-fucose-deficient version of rat-human chimeric anti-human PDPN mAb (NZ-12), named as NZ-12f, and compared their binding affinity, ADCC/CDC activities, and antitumor activity.

\section{Materials and Methods}

\section{Cell lines}

LN319 was purchased from Addexbio Technologies (San Diego, CA). ExpiCHO-S was purchased from Thermo Fisher Scientific, Inc., (Waltham, MA). BINDS-09 (FUT8-knocked out ExpiCHO-S cells) was established in our previous study. ${ }^{(36)}$ LN319 was cultured in Dulbecco's modified Eagle's medium (DMEM) medium (Nacalai Tesque, Inc., Kyoto, Japan), supplemented with $10 \%$ heat-inactivated fetal bovine serum (Thermo Fisher Scientific, Inc.) at $37^{\circ} \mathrm{C}$ in a humidified atmosphere of $5 \% \mathrm{CO}_{2}$ and $95 \%$ air. ExpiCHO-S and BINDS09 were cultured following manufacturer's instructions.

\section{Antibodies}

For the generation of a rat-human chimeric anti-human PDPN mAb (NZ-12), appropriate $\mathrm{V}_{\mathrm{H}}$ of rat NZ-1 antibody and $\mathrm{C}_{\mathrm{H}}$ of human $\mathrm{IgG}_{1}$ were subcloned into pCAG-Neo (FUJIFILM Wako Pure Chemical Corporation, Osaka, Japan), and $\mathrm{V}_{\mathrm{L}}$ of rat NZ-1 antibody and $\mathrm{C}_{\mathrm{L}}$ of human lambda chain were subcloned into pCAG-Ble vectors (FUJIFILM Wako Pure Chemical Corporation). For creation of a humanized antihuman PDPN mAb (NZ-27), the complementarity determining region $(\mathrm{CDR})$ of $\mathrm{V}_{\mathrm{H}}$ in the NZ-1 antibody, frame sequences of $\mathrm{V}_{\mathrm{H}}$ in human $\mathrm{Ig}$, and $\mathrm{C}_{\mathrm{H}}$ of human $\mathrm{IgG}_{1}$ were subcloned into the pCAG-Neo vector (FUJIFILM Wako Pure Chemical Corporation). The CDR of $\mathrm{V}_{\mathrm{L}}$ in the NZ-1 antibody, frame sequences of $\mathrm{V}_{\mathrm{L}}$ in human $\mathrm{Ig}$, and $\mathrm{C}_{\mathrm{L}}$ of human lambda chain were subcloned into the pCAG-Ble vector (FUJIFILM Wako Pure Chemical Corporation). Antibody expression vectors of NZ-27 and NZ-12 were transfected into BINDS-09 using the ExpiCHO-S Expression System (Thermo Fisher Scientific, Inc.), respectively. Produced mAbs are named as P1027 and NZ-12f, respectively. P1027 and NZ-12f were purified using Protein G Sepharose 4 Fast Flow (GE Healthcare UK, Ltd., Buckinghamshire, England). Human IgG was purchased from Sigma-Aldrich Corp. (St. Louis, MO).

\section{Determination of the binding affinity by flow cytometry}

LN319 cells $\left(2 \times 10^{5}\right.$ cells) were resuspended with $100 \mu \mathrm{L}$ of serially diluted antibodies (P1027 and NZ-12f; 0.006$100 \mu \mathrm{g} / \mathrm{mL}$ ) followed by FITC-labeled anti-human IgG (Thermo Fisher Scientific, Inc.). Fluorescence data were collected using a cell analyzer (EC800; Sony Corp., Tokyo, Japan). Dissociation constants $\left(K_{\mathrm{D}}\right)$ were obtained by fitting binding isotherms using built-in one-site binding models in GraphPad Prism 6 (GraphPad Software, Inc., La Jolla, CA).

\section{ADCC reporter gene assay}

We performed the Fc $\gamma$ RIIIa stimulation assay using an ADCC Reporter Bioassay kit from Promega (Madison, WI), following the manufacturer's instructions. ${ }^{(37)}$ LN319 was inoculated into a 96-well white solid plate, 12,500 cells per well. Anti-PDPN mAbs were serially diluted and added to LN319 cells. Engineered, immortalized Jurkat T lymphocytes (Promega) expressing the Fc $\gamma$ RIIIa receptor $(75,000$ cells in $25 \mu \mathrm{L}$ ) were then added and cocultured with antibodytreated target cells at $37^{\circ} \mathrm{C}$ for $6 \mathrm{~h}$. Luminescence using the Bio-Glo Luciferase Assay System (Promega) was measured with a GloMax luminometer (Promega).

\section{Atibody-dependent cellular cytotoxicity}

Human NK cells were purchased from Takara Bio, Inc., (Shiga, Japan). Target cells were labeled with $10 \mu \mathrm{g} / \mathrm{mL}$ Calcein AM (Thermo Fisher Scientific, Inc.) and resuspended in the same medium. Target cells were plated in 96-well plates $\left(1 \times 10^{4}\right.$ cells/well $)$ and mixed with effector cells, antiPDPN antibodies, or control human IgG. After a 6.5-hour incubation, Calcein AM release to the supernatant from each well was measured. Fluorescence intensity was determined using a microplate reader (Power Scan HT; BioTek Instruments, Winooski, VT) with excitation and emission wavelengths of 485 and $538 \mathrm{~nm}$, respectively. Cytolytic activity (\% of lysis) was calculated as \% lysis $=(E-S) /(M-S) \times 100$, where $E$ is the fluorescence of combined target and effector cells, $S$ is spontaneous fluorescence of target cells only, and $M$ is maximum fluorescence measured after lysing all cells with a buffer containing $0.5 \%$ Triton X-100, $10 \mathrm{mM}$ Tris- $\mathrm{HCl}$ (pH 7.4), and $10 \mathrm{mM}$ of EDTA.

\section{Complement-dependent cytotoxicity}

Target cells were labeled with $10 \mu \mathrm{g} / \mathrm{mL}$ Calcein AM and resuspended in the same medium. The target cells were plated 
in 96-well plates $\left(2 \times 10^{4}\right.$ cells/well $)$ and mixed with either anti-PDPN antibodies or control human $\operatorname{IgG}$ and $10 \%$ of rabbit complement. After a 6.5-hour incubation, the Calcein AM release of supernatant from each well was measured. Fluorescence intensity was determined using a microplate reader (Power Scan HT) with an excitation wavelength of $485 \mathrm{~nm}$ and an emission wavelength of $538 \mathrm{~nm}$. Cytolytic activity (\% of lysis) was calculated as \% lysis $=(E-S)$ / $(M-S) \times 100$, where $E$ is fluorescence of combined target and effector cells, $S$ is spontaneous fluorescence of target cells only, and $M$ is maximum fluorescence measured after lysing all cells with a buffer containing $0.5 \%$ Triton X-100, $10 \mathrm{mM}$ Tris- $\mathrm{HCl}$ (pH 7.4), and $10 \mathrm{mM}$ of EDTA.

\section{Antitumor activity of anti-PDPN antibodies} in xenografts of LN319

Sixteen 6-week-old female BALB/c nude mice were purchased from Charles River and used at 7 weeks of age. LN319 cells $\left(0.3 \mathrm{~mL}\right.$ of $1.33 \times 10^{8}$ cells $/ \mathrm{mL}$ in DMEM) were mixed with $0.5 \mathrm{~mL}$ BD Matrigel Matrix Growth Factor Reduced (BD Biosciences, San Jose, CA). Mice were injected subcutaneously in the left flank $(100 \mu \mathrm{L})$ with the suspension mentioned earlier $\left(5 \times 10^{6}\right.$ cells $)$. After day $1,100 \mu \mathrm{g}$ of P1027, NZ-12f, and control human IgG in $100 \mu \mathrm{L}$ PBS were injected into mice through intraperitoneal injection (i.p.). Additional antibodies were injected on days 8 and 16. Human
NK cells $\left(8.0 \times 10^{5}\right.$ cells $)$ were injected around the tumors on days 1,8 , and 16 . Twenty-one days after cell implantation, all mice were euthanized by cervical dislocation and tumor diameters and volumes were determined as previously described. ${ }^{(38)}$

\section{Statistical analyses}

All data were expressed as mean \pm SEM. Statistical analysis used ANOVA and Tukey-Kramer's test using R statistical (R Foundation for Statistical Computing, Vienna, Austria). $p<0.05$ was adopted as a level of statistical significance.

\section{Results}

Development of a core-fucose-deficient humanized anti-PDPN $m A b$ (P1027) and a core-fucose-deficient rat-human chimeric $m A b$ (NZ-12f)

In this study, we produced core-fucose-deficient humanized anti-PDPN (P1027) and rat-human chimeric antibodies (NZ-12f) (Fig. 1). Binding affinity of P1027 and NZ-12f against LN319 cells was $1.1 \times 10^{-8}$ and $3.9 \times 10^{-9} \mathrm{M}$, respectively, indicating that both mAbs possess high affinity for LN319 cells, but NZ-12f possesses higher binding activity (Fig. 2).

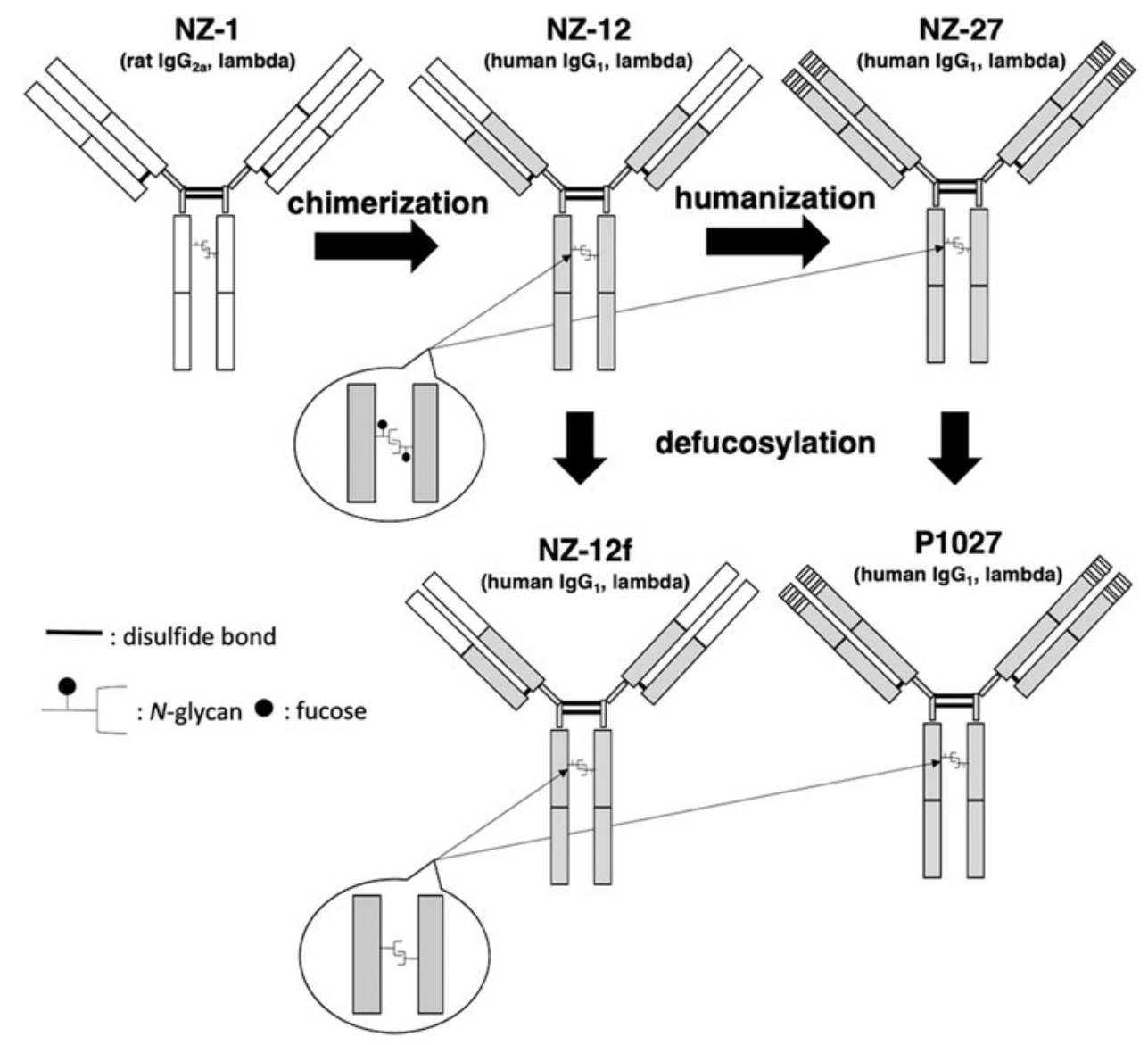

FIG. 1. Development of a core-fucose-deficient humanized anti-PDPN mAb (P1027) and a core-fucose-deficient rathuman chimeric mAb (NZ-12f). White box, derived from NZ-1 (rat IgG); gray box, derived from human IgG. $\mathrm{mAb}$, monoclonal antibody; PDPN, podoplanin. 

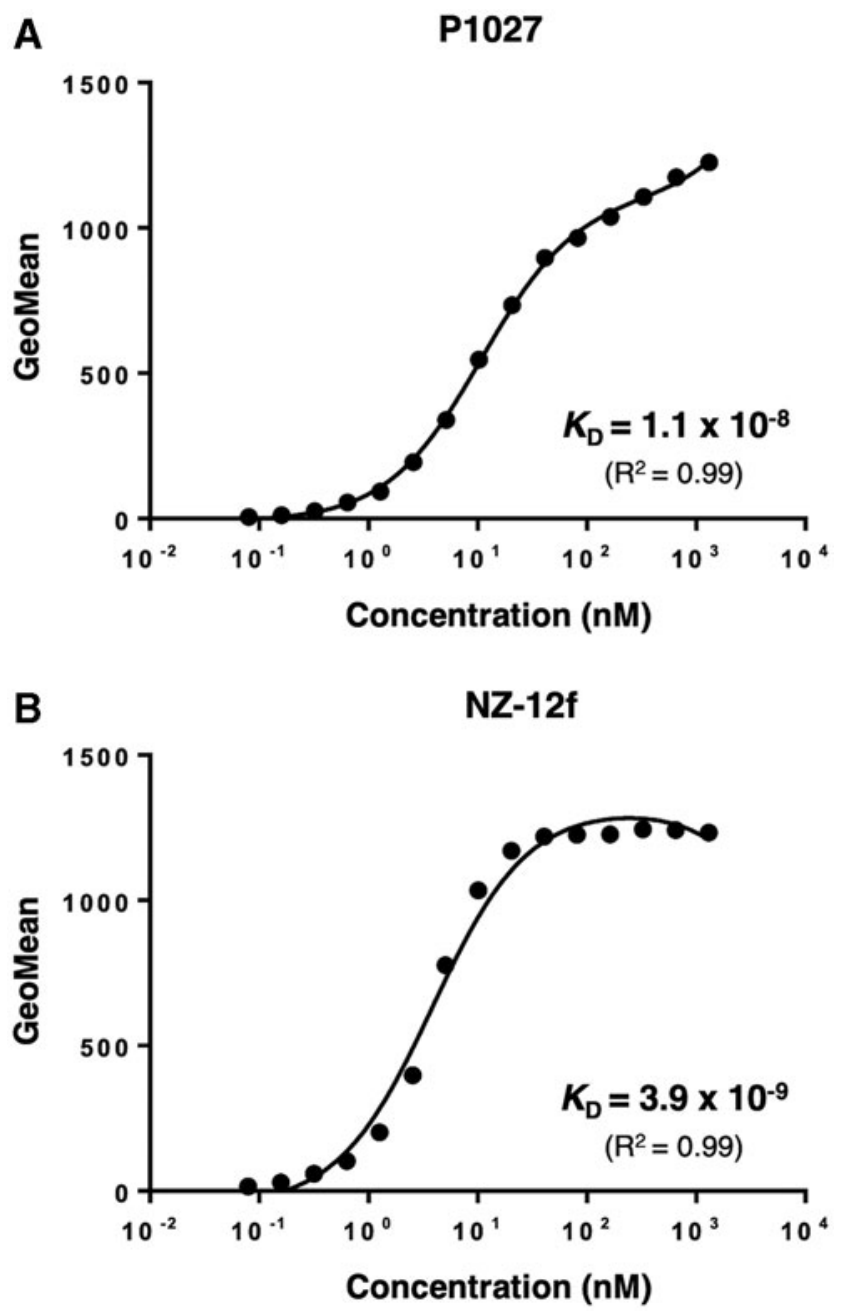

FIG. 2. Determination of binding affinity using flow cytometry. LN319 $\left(2 \times 10^{5}\right.$ cells $)$ was resuspended with $100 \mu \mathrm{L}$ of serially diluted P1027 (A) or NZ-12f (B) $(0.006-100 \mu \mathrm{g} / \mathrm{mL})$.

\section{$A D C C$ reporter gene assay}

We used LN319 glioblastoma cells as target cells, and compared luminescence between P1027 and NZ-12f. EC50s for P1027 and NZ-12f were 149 and $14.3 \mathrm{ng} / \mathrm{mL}$, respectively (Fig. 3A, B). At a concentration of $3.3 \mu \mathrm{g} / \mathrm{mL}, \mathrm{NZ}-12 \mathrm{f}$ showed 1.5 times higher luminescence than P1027 (Fig. 3C). Thus, NZ-12f might display higher ADCC activity than P1027.

\section{ADCC and CDC mediated by anti-PDPN antibodies}

To apply targeted therapy toward PDPN, we investigated ADCC induction against PDPN-expressing cell lines by antiPDPN antibodies using human NK cells as effector cells. NZ-12f exhibited ADCC activity (51\% cytotoxicity, Fig. 4A) and CDC activity (58\% cytotoxicity, Fig. 4B) against LN319 cells; P1027 exhibited ADCC activity (23\% cytotoxicity, Fig. 4A) and CDC activity (53\% cytotoxicity, Fig. 4B) against LN319 cells, indicating that both NZ-12f and P1027 showed high CDC activities against LN319 cells (P1027 vs. control, $p<0.01$; NZ-12f vs. control, $p<0.01$; P1027 vs. NZ-12f, n.s.), whereas NZ-12f possesses 2.2 times higher
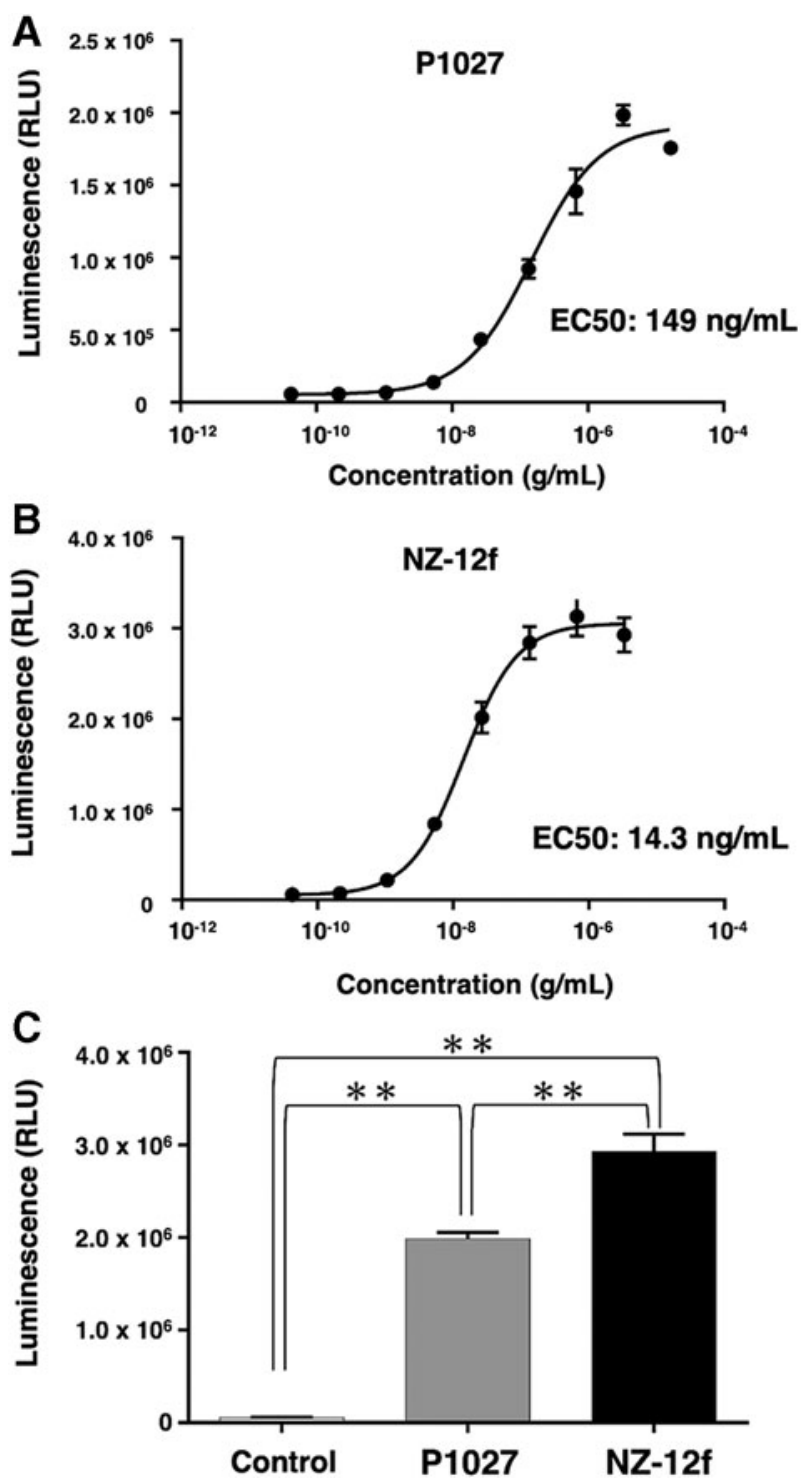

FIG. 3. ADCC bioreporter assay. (A, B) Dose dependency of anti-PDPN mAbs in the ADCC reporter assay. P1027 (A) and NZ-12f (B) were serially diluted and incubated with the LN319 cells. As effector cells, engineered, immortalized Jurkat T lymphocytes were cocultured. Luminescence using a Bio-Glo Luciferase Assay System was measured with a GloMax luminometer. (C) Comparison of luminescence between P1027 and NZ-12f in the ADCC bioreporter assay at a concentration of $3.3 \mu \mathrm{g} / \mathrm{mL}$. Asterisks indicate statistical significance $(* * p<0.01$, Tukey-Kramer's test). ADCC, antibodydependent cellular cytotoxicity.

ADCC compared with P1027 (P1027 vs. control, n.s.; NZ-12f vs. control, $p<0.01 ; \mathrm{P} 1027$ vs. NZ-12f, $p<0.01)$.

\section{Antitumor activity of $P 1027$ and NZ-12f} in LN319 xenografts

Induction of cytotoxicity by P1027 and NZ-12f was investigated in vivo using LN319 cell xenografts in the flanks of nude mice. P1027, NZ-12f, and control human IgG were injected i.p. three times, on days 1, 8, and 16 after cell injection. Human NK cells were injected around tumors on days 1,8 , and 16 . Tumor formation was observed in mice 

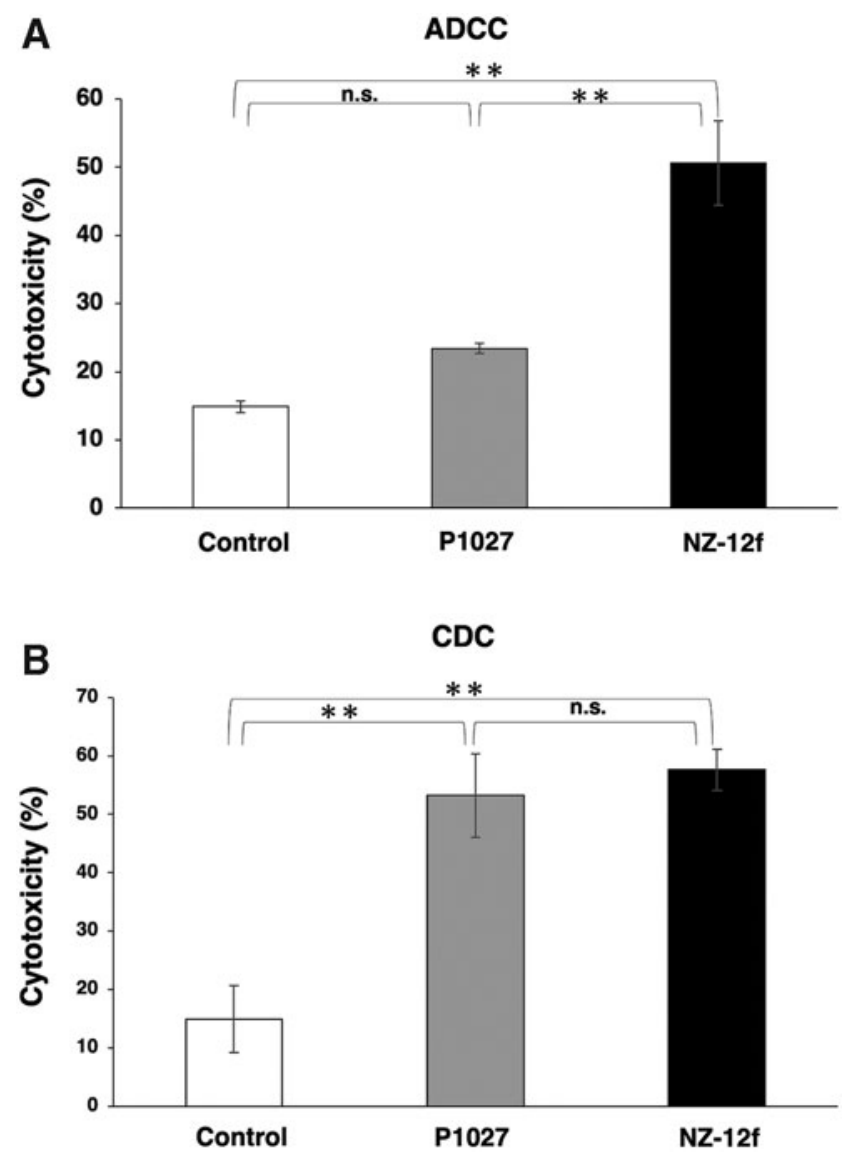

FIG. 4. ADCC and CDC activities of anti-PDPN antibodies. (A) ADCC activities induced by human NK cells against LN319 were determined. (B) CDC activities against LN319 were determined. Asterisks indicate statistical significance $(* * p<0.01$, n.s.: not significant, Tukey-Kramer's test). $\mathrm{CDC}$, complement-dependent cytotoxicity; NK, natural killer.

in every group. P1027 and NZ-12f treatment significantly reduced tumor development compared with control mice on days 3, 7, 10, 13, 16, and 21 (Fig. 5). No difference between P1027 and NZ-12f was observed at any time. Weights of tumors from P1027 and NZ-12f-treated mice were significantly less than for tumors from IgG-treated control mice (Fig. 6A). No difference in weights of tumors between P1027 and NZ-12f was found. Resected tumors are depicted in Figure 6B. Total body weight was not significantly different among the three groups (Fig. 7A). Mice on day 21 are depicted in Figure 7B. Both P1027 and NZ-12f exert a significant antitumor effect against PDPN-expressing brain tumors.

\section{Discussion}

We previously produced a FUT8-knockout cell line (BINDS-09) from ExpiCHO-S cells. ${ }^{(36)}$ By using BINDS-09, we easily produced core-fucose-deficient antibodies to enhance ADCC activity. In this study, we produced both a corefucose-deficient humanized anti-PDPN antibody (P1027) and a rat-human chimeric antibody (NZ-12f). Binding affinities of P1027 and NZ-12f against LN319 cells were $1.1 \times 10^{-8}$ and $3.9 \times 10^{-9} \mathrm{M}$ (Fig. 2), of the affinity of NZ-12f against LN319 is 2.8 times than the affinity of P1027. We next investigated whether this difference of binding affinity affects

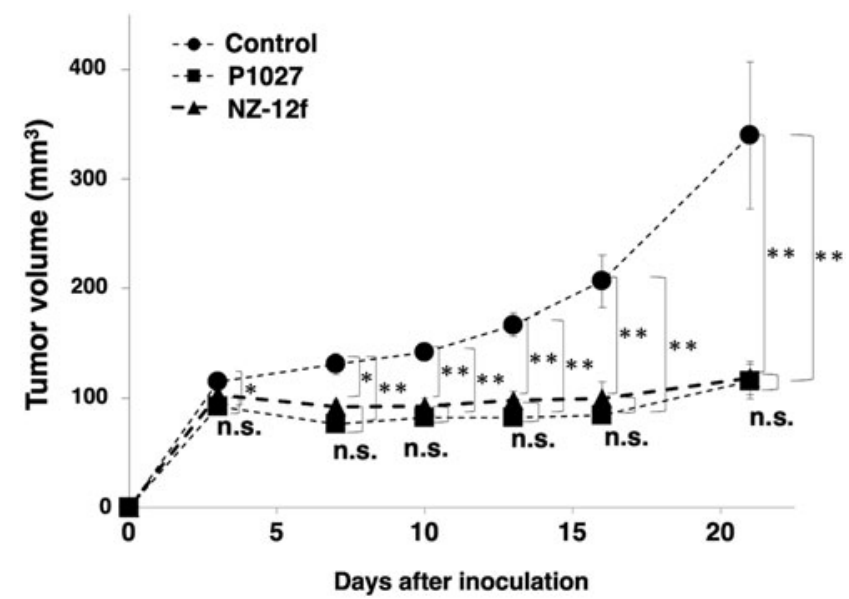

FIG. 5. Evaluation of antitumor activity of anti-PDPN antibodies (tumor volume). Tumor volume was measured in mice with LN319 xenografts. Asterisks indicate statistical significance $(* p<0.05, * * p<0.01$, n.s.: not significant, TukeyKramer's test).

the ability of antibodies to activate the ADCC reporter gene. The ADCC reporter system consists of an $\mathrm{Fc}$ region of an antibody that binds to the Fc $\gamma$ RIIIa receptor expressed on an engineered immortalized Jurkat T-lymphocyte cell line. ${ }^{(37)}$ This binding activates gene transcription through the nuclear factor of the activated $\mathrm{T}$ cell pathway, inducing the expression of firefly luciferase. The Fc $\gamma$ RIIIa stimulation assay can be used to evaluate the ability of an antibody to activate the

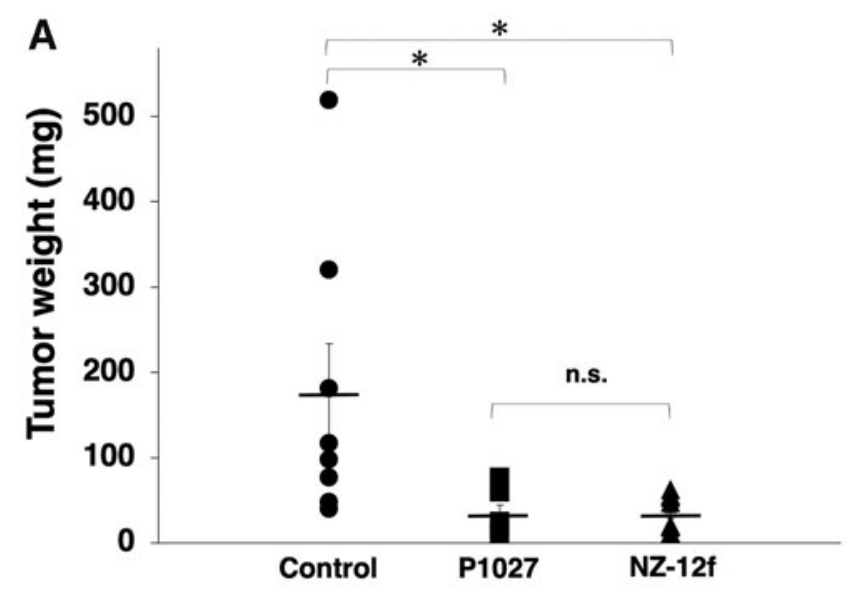

B

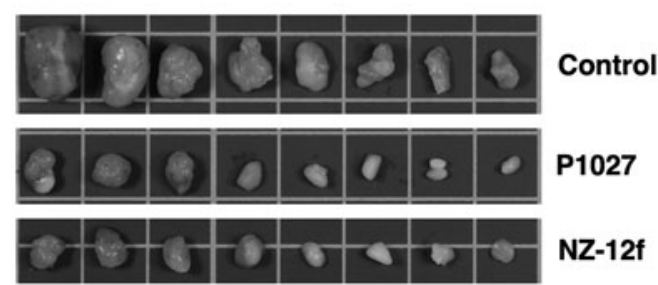

FIG. 6. Evaluation of antitumor activity of anti-PDPN antibodies (tumor weight). (A) Tumor weight was measured from excised LN319 xenografts. (B) Resected tumors of LN319 xenografts on day 21. Values are mean \pm SEM. An asterisk indicates statistical significance $(* p<0.05$, n.s.: not significant, Tukey-Kramer's test). 


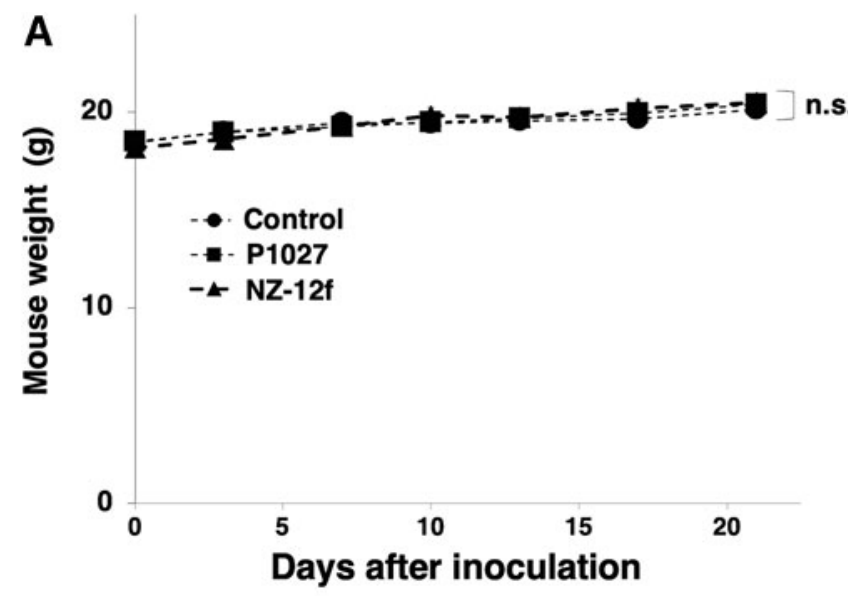

B

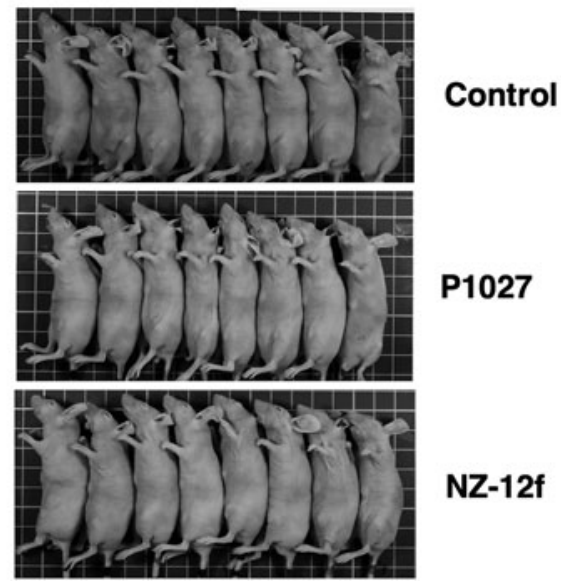

FIG. 7. Evaluation of antitumor activity of anti-PDPN antibodies (body weight). (A) Body weights of mice with LN319 xenografts. n.s.: not significant. (B) Appearance of treated mice on day 21 .

ADCC reporter gene. NZ-12f displays 1.5 times higher activation of the ADCC reporter gene compared with P1027 (Fig. 3), indicating that binding affinity is associated with the ability of antibodies to activate the ADCC reporter gene. In the ADCC and CDC assays, both P1027 and NZ-12f demonstrated high CDC activities, whereas NZ-12f showed 2.2 times higher ADCC activity compared with P1027 (Fig. 4), which are compatible with results from binding affinity and ADCC reporter assays.

In our previous studies, antitumor effects were observed after treatment of malignant pleural mesothelioma orthotopic xenograft mice with NZ-12 and human NK cells. ${ }^{(31)}$ Furthermore, RIT with ${ }^{90} \mathrm{Y}$-labeled NZ-12 significantly suppressed tumor growth in an NCI-H226 xenograft mouse model, and prolonged survival without obvious adverse effects. ${ }^{(32)}$ Although P1027 showed lower binding affinity and lower ADCC activities to LN319 cells, antitumor activity of P1027 for LN319 xenografts was equal to that of NZ-12f (Figs. 5 and 6). We further conducted additional in vivo experiments using three times injection of $10 \mu \mathrm{g}$ antibodies to assess whether the antitumor activities were saturated when three times injection of $100 \mu \mathrm{g}$ was employed. However, we observed no difference of antitumor activities between P1027 and NZ-12f using three times injection of $10 \mu \mathrm{g}$ antibodies (data not shown), suggesting that antitumor activities were exerted mainly by CDC because both P1027 and NZ-12f showed high CDC activities against LN319 cells (Fig. 4B). We need to further perform in vivo experiments using the other xenograft models, such as lung cancers and malignant mesotheliomas to confirm that humanized anti-PDPN mAbs may be effective for patients with PDPN-expressing cancers.

\section{Acknowledgment}

We thank Ms. Akiko Harakawa for technical assistance in animal experiments.

\section{Author Disclosure Statement}

No competing financial interests exist.

\section{Funding Information}

This research was supported in part by AMED under Grant Numbers: JP20am0401013 (Y.K.), JP20am0101078 (Y.K.), JP20ae0101028 (Y.K.), and JP20bm1004001 (Y.K.), and by JSPS KAKENHI Grant Numbers 20K16322 (M.S.), 17K07299 (M.K.K.), and 19K07705 (Y.K.).

\section{References}

1. Kato Y, Fujita N, Kunita A, Sato S, Kaneko M, Osawa M, and Tsuruo T: Molecular identification of Aggrus/T1alpha as a platelet aggregation-inducing factor expressed in colorectal tumors. J Biol Chem 2003;278:51599-51605.

2. Breiteneder-Geleff S, Soleiman A, Kowalski H, Horvat R, Amann G, Kriehuber E, Diem K, Weninger W, Tschachler E, Alitalo K, and Kerjaschki D: Angiosarcomas express mixed endothelial phenotypes of blood and lymphatic capillaries: Podoplanin as a specific marker for lymphatic endothelium. Am J Pathol 1999;154:385-394.

3. Kato Y, Sasagawa I, Kaneko M, Osawa M, Fujita N, and Tsuruo T: Aggrus: A diagnostic marker that distinguishes seminoma from embryonal carcinoma in testicular germ cell tumors. Oncogene 2004;23:8552-8556.

4. Kato Y, Kaneko M, Sata M, Fujita N, Tsuruo T, and Osawa M: Enhanced expression of Aggrus (T1alpha/podoplanin), a platelet-aggregation-inducing factor in lung squamous cell carcinoma. Tumor Biol 2005;26:195-200.

5. Kato Y, Kaneko MK, Kuno A, Uchiyama N, Amano K, Chiba Y, Hasegawa Y, Hirabayashi J, Narimatsu H, Mishima K, and Osawa M: Inhibition of tumor cell-induced platelet aggregation using a novel anti-podoplanin antibody reacting with its platelet-aggregation-stimulating domain. Biochem Biophys Res Commun 2006;349:1301-1307.

6. Kimura N, and Kimura I: Podoplanin as a marker for mesothelioma. Pathol Int 2005;55:83-86.

7. Mishima K, Kato Y, Kaneko MK, Nakazawa Y, Kunita A, Fujita N, Tsuruo T, Nishikawa R, Hirose T, and Matsutani M: Podoplanin expression in primary central nervous system germ cell tumors: A useful histological marker for the diagnosis of germinoma. Acta Neuropathol (Berl) 2006; 111:563-568.

8. Mishima K, Kato Y, Kaneko MK, Nishikawa R, Hirose T, and Matsutani $\mathrm{M}$ : Increased expression of podoplanin in malignant astrocytic tumors as a novel molecular marker of malignant progression. Acta Neuropathol (Berl) 2006;111: 483-488. 
9. Kunita A, Kashima TG, Ohazama A, Grigoriadis AE, and Fukayama M: Podoplanin is regulated by AP-1 and promotes platelet aggregation and cell migration in osteosarcoma. Am J Pathol 2011;179:1041-1049.

10. Gandarillas A, Scholl FG, Benito N, Gamallo C, and Quintanilla M: Induction of PA2.26, a cell-surface antigen expressed by active fibroblasts, in mouse epidermal keratinocytes during carcinogenesis. Mol Carcinog 1997;20: $10-18$.

11. Sugimoto $Y$, Watanabe M, Oh-hara T, Sato S, Isoe T, and Tsuruo T: Suppression of experimental lung colonization of a metastatic variant of murine colon adenocarcinoma 26 by a monoclonal antibody $8 \mathrm{~F} 11$ inhibiting tumor cell-induced platelet aggregation. Cancer Res 1991;51:921-925.

12. Kato $\mathrm{Y}$, Kaneko MK, Kunita A, Ito H, Kameyama A, Ogasawara S, Matsuura N, Hasegawa Y, Suzuki-Inoue K, Inoue O, Ozaki Y, and Narimatsu H: Molecular analysis of the pathophysiological binding of the platelet aggregationinducing factor podoplanin to the C-type lectin-like receptor CLEC-2. Cancer Sci 2008;99:54-61.

13. Kunita A, Kashima TG, Morishita Y, Fukayama M, Kato Y, Tsuruo T, and Fujita N: The platelet aggregationinducing factor aggrus/podoplanin promotes pulmonary metastasis. Am J Pathol 2007;170:1337-1347.

14. Martin-Villar E, Megias D, Castel S, Yurrita MM, Vilaro S, and Quintanilla M: Podoplanin binds ERM proteins to activate RhoA and promote epithelial-mesenchymal transition. J Cell Sci 2006;119:4541-4553.

15. Hoshino A, Ishii G, Ito T, Aoyagi K, Ohtaki Y, Nagai K, Sasaki H, and Ochiai A: Podoplanin-positive fibroblasts enhance lung adenocarcinoma tumor formation: Podoplanin in fibroblast functions for tumor progression. Cancer Res 2011;71:4769-4779.

16. Kono T, Shimoda M, Takahashi M, Matsumoto K, Yoshimoto T, Mizutani M, Tabata C, Okoshi K, Wada H, and Kubo H: Immunohistochemical detection of the lymphatic marker podoplanin in diverse types of human cancer cells using a novel antibody. Int J Oncol 2007;31:501-508.

17. Roy S, Chu A, Trojanowski JQ, and Zhang PJ: D2-D40, a novel monoclonal antibody against the M2A antigen as a marker to distinguish hemangioblastomas from renal cell carcinomas. Acta Neuropathol (Berl) 2005;109:497-502.

18. Scholl FG, Gamallo C, and Quintanilla M: Ectopic expression of PA2.26 antigen in epidermal keratinocytes leads to destabilization of adherens junctions and malignant progression. Lab Invest 2000;80:1749-1759.

19. Yuan P, Temam S, El-Naggar A, Zhou X, Liu D, Lee J, and Mao L: Overexpression of podoplanin in oral cancer and its association with poor clinical outcome. Cancer 2006; 107:563-569.

20. Kawaguchi H, El-Naggar AK, Papadimitrakopoulou V, Ren H, Fan YH, Feng L, Lee JJ, Kim E, Hong WK, Lippman SM, and Mao L: Podoplanin: A novel marker for oral cancer risk in patients with oral premalignancy. J Clin Oncol 2008;26:354-360.

21. Kawase A, Ishii G, Nagai K, Ito T, Nagano T, Murata Y, Hishida T, Nishimura M, Yoshida J, Suzuki K, and Ochiai A: Podoplanin expression by cancer associated fibroblasts predicts poor prognosis of lung adenocarcinoma. Int $\mathrm{J}$ Cancer 2008;123:1053-1059.

22. Singh SK, Hawkins C, Clarke ID, Squire JA, Bayani J, Hide T, Henkelman RM, Cusimano MD, and Dirks PB: Identification of human brain tumour initiating cells. Nature 2004;432:396-401.
23. Bao S, Wu Q, McLendon RE, Hao Y, Shi Q, Hjelmeland AB, Dewhirst MW, Bigner DD, and Rich JN: Glioma stem cells promote radioresistance by preferential activation of the DNA damage response. Nature 2006;444:756-760.

24. Atsumi N, Ishii G, Kojima M, Sanada M, Fujii S, and Ochiai A: Podoplanin, a novel marker of tumor-initiating cells in human squamous cell carcinoma A431. Biochem Biophys Res Commun 2008;373:36-41.

25. Fujii Y, Kaneko M, Neyazaki M, Nogi T, Kato Y, and Takagi J: PA tag: A versatile protein tagging system using a super high affinity antibody against a dodecapeptide derived from human podoplanin. Protein Expr Purif 2014;95: 240-247.

26. Fujii Y, Matsunaga Y, Arimori T, Kitago Y, Ogasawara S, Kaneko MK, Kato Y, and Takagi J: Tailored placement of a turn-forming PA tag into the structured domain of a protein to probe its conformational state. J Cell Sci 2016;129: $1512-1522$.

27. Kato Y, Vaidyanathan G, Kaneko MK, Mishima K, Srivastava N, Chandramohan V, Pegram C, Keir ST, Kuan CT, Bigner DD, and Zalutsky MR: Evaluation of antipodoplanin rat monoclonal antibody NZ-1 for targeting malignant gliomas. Nucl Med Biol 2010;37:785-794.

28. Chandramohan V, Bao X, Kato Kaneko M, Kato Y, Keir ST, Szafranski SE, Kuan CT, Pastan IH, and Bigner DD: Recombinant anti-podoplanin (NZ-1) immunotoxin for the treatment of malignant brain tumors. Int J Cancer 2013; 132:2339-2348.

29. Kaneko MK, Kunita A, Abe S, Tsujimoto Y, Fukayama M, Goto K, Sawa Y, Nishioka Y, and Kato Y: Chimeric antipodoplanin antibody suppresses tumor metastasis through neutralization and antibody-dependent cellular cytotoxicity. Cancer Sci 2012;103:1913-1919.

30. Kaneko MK, Abe S, Ogasawara S, Fujii Y, Yamada S, Murata T, Uchida H, Tahara H, Nishioka Y, and Kato Y: Chimeric anti-human podoplanin antibody NZ-12 of lambda light chain exerts higher antibody-dependent cellular cytotoxicity and complement-dependent cytotoxicity compared with NZ-8 of kappa light chain. Monoclon Antib Immunodiagn Immunother 2017;36:25-29.

31. Abe S, Kaneko MK, Tsuchihashi Y, Izumi T, Ogasawara S, Okada N, Sato C, Tobiume M, Otsuka K, Miyamoto L, Tsuchiya K, Kawazoe K, Kato Y, and Nishioka Y: Antitumor effect of novel anti-podoplanin antibody NZ-12 against malignant pleural mesothelioma in an orthotopic xenograft model. Cancer Sci 2016;107:1198-1205.

32. Sudo H, Tsuji AB, Sugyo A, Saga T, Kaneko MK, Kato Y, and Higashi T: Therapeutic efficacy evaluation of radioimmunotherapy with (90) Y-labeled anti-podoplanin antibody NZ-12 for mesothelioma. Cancer Sci 2019;110: 1653-1664.

33. Niwa R, Shoji-Hosaka E, Sakurada M, Shinkawa T, Uchida K, Nakamura K, Matsushima K, Ueda R, Hanai N, and Shitara K: Defucosylated chimeric anti-CC chemokine receptor 4 IgG1 with enhanced antibody-dependent cellular cytotoxicity shows potent therapeutic activity to T-cell leukemia and lymphoma. Cancer Res 2004;64:21272133.

34. Shinkawa T, Nakamura K, Yamane N, Shoji-Hosaka E, Kanda Y, Sakurada M, Uchida K, Anazawa H, Satoh M, Yamasaki M, Hanai N, and Shitara K: The absence of fucose but not the presence of galactose or bisecting $\mathrm{N}$-acetylglucosamine of human IgG1 complex-type oligosaccharides shows the critical role of enhancing antibody- 
dependent cellular cytotoxicity. J Biol Chem 2003;278: 3466-3473.

35. Itai S, Ohishi T, Kaneko MK, Yamada S, Abe S, Nakamura T, Yanaka M, Chang YW, Ohba SI, Nishioka Y, Kawada M, Harada $\mathrm{H}$, and Kato Y: Anti-podocalyxin antibody exerts antitumor effects via antibody-dependent cellular cytotoxicity in mouse xenograft models of oral squamous cell carcinoma. Oncotarget 2018;9:22480-22497.

36. Kato Y, Mizuno T, Yamada S, Nakamura T, Itai S, Yanaka M, Sano M, and Kaneko MK: Establishment of P38Bf, a core-fucose-deficient mouse-canine chimeric antibody against dog podoplanin monoclon. Antib Immunodiagn Immunother 2018;37:218-223.

37. Parekh BS, Berger E, Sibley S, Cahya S, Xiao L, LaCerte MA, Vaillancourt P, Wooden S, and Gately D: Development and validation of an antibody-dependent cellmediated cytotoxicity-reporter gene assay. MAbs 2012;4: $310-318$.
38. Kato Y, Kunita A, Abe S, Ogasawara S, Fujii Y, Oki H, Fukayama M, Nishioka Y, and Kaneko MK: The chimeric antibody chLpMab-7 targeting human podoplanin suppresses pulmonary metastasis via $\mathrm{ADCC}$ and $\mathrm{CDC}$ rather than via its neutralizing activity. Oncotarget 2015;6:36003-36018.

Address correspondence to: Yukinari Kato New Industry Creation Hatchery Center Tohoku University 2-1, Seiryo-machi, Aoba-ku Sendai 980-8575

Japan

E-mail: yukinarikato@med.tohoku.ac.jp

Received: April 17, 2020 Accepted: August 20, 2020 